\title{
Carbon Nanofiber-Based Wearable Patches for Bio-Potential Monitoring
}

\author{
Hachul Jung ${ }^{1}$ (1) $\cdot$ Dahye Kwon ${ }^{1} \cdot$ Seung-A Lee ${ }^{1}$ Young-Jin Kim ${ }^{1} \cdot$ Jin Woo Ahn ${ }^{1}$
}

Received: 22 October 2018 / Accepted: 22 April 2019 / Published online: 25 April 2019

(c) The Author(s) 2019

\begin{abstract}
Purpose We fabricated a wearable patch including a novel patch-type flexible dry electrode based on carbon nanofibers (CNFs) and biocompatible PDMS (MED-6215) for long-term bio-potential monitoring.

Methods The patch comprises dry electrode parts for recording bio signals and sticky parts for skin mounting. To evaluate electrical characteristics and electrocardiograph (ECG) recording, the CNF concentrations and dry electrode thickness were varied. The electromechanical properties of the dry electrode were tested under cyclic load conditions. Cytotoxicity tests and long-term wearing tests were conducted to prove biocompatibility.

Results The fabricated wearable patch is flexible, stretchable, easily skin mountable, and directly connectable with the measuring system. CNF concentrations is the most important variable compared with diameter, thickness for obtaining high-quality ECG signals. The electromechanical tests revealed excellent recovery performances in resistivity and durability. Cytotoxicity tests showed no effects on cells and long-term wearing tests showed no skin reactions.

Conclusions The fabricated patch can be utilized for long-term bio-potential monitoring such as electromyography and electroencephalograms. The proposed dry electrode has flexibility, conductivity, and biocompatibility and is widely applicable to wearable sensors.
\end{abstract}

Keywords Carbon nanofibers $\cdot$ Electrocardiograph $\cdot$ Wearable $\cdot$ Dry electrode

\section{Introduction}

Recently, the wearable devices market has grown rapidly. Healthcare applications receive particular focus because many people want to be able to monitor their physical condition anywhere and at any time [1-14]. For successful monitoring with no skin irritation, long term wearable biocompatible electrodes are critical and electrocardiography is a key bio signal [15]. However, there are many remaining issues related to the battery, miniaturization, weight, and long-term biocompatibility.

The latest research solves these issues by recording bio-signals without the use of additive gels. Conventional electrodes are wet electrodes that include gels to improve

Hachul Jung

hacher99@kbiohealth.kr

1 Integrated Medical Technology Team, Department of Research and Development, Medical Device Development Center, Osong Medical Innovation Foundation, Cheongju-si, Republic of Korea adhesion onto the skin and resist external environmental factors; however, with time, dehydration of the gel causes decreasing signal quality, increasing signal noise, and skin irritation. Extensive research is attempting to solve these issues [2, 4, 6, 16-23].

In this study, we propose a carbon nanofiber (CNF)-based wearable patch that is directly connectable to conventional electrocardiograph (ECG) measuring devices. It is fabricated by assembling dry electrode and sticky patch components. CNFs are dispersed in a Polydimethylsiloxane (PDMS) matrix in order to develop a flexible nano-composite material as an electrode. PDMS, a well-known superior elastic, is flexible, has optically transparent properties, and has recently become a popular choice for biomedical applications because of its non- toxicity, high gas-permeability, and amenable fabric ability [24, 25]. Sylgard-184 is the most used PDMS in these applications but its biocompatibility must be evaluated because it was developed specifically for electronic and lighting uses. MED-6215 was developed for medical and implantable PDMS uses and is considered 
acceptable for human implantation for periods greater than 29 days according to biocompatibility tests [26, 27].

Conversely, carbon-based nanomaterials such as carbon nanotubes (CNTs) and CNFs show excellent mechanical, electrical, and thermal properties and are inert in the context of biomedical applications.

For these reasons, these materials have been widely used in a variety of applications. However, CNTs have questionable biocompatibility with the human body $[28,29]$ and CNFs are more easily uniformly dispersed in elastomer than CNTs because of aggregation conditions [30-33].

In this study, the dispersion protocol and concentrations were parametrized in order to achieve very good dispersion. To optimize electrical performance and the diffusion degree of uniformity, we developed a unique mixing and baking process. Secondly, sticky patch parts were made by patterning and detaching from a smooth surface substrate after spincoating soft skin adhesive. In this process, the attachable and detachable strengths of the sticky patch are measured and optimized for use in a monitoring system. The electrical properties and biocompatibilities of the wearable patch were evaluated and ECG signals were measured by changing the CNF ratio and the thickness of the electrode.

\section{Materials and Methods}

\subsection{Materials}

CNFs (GNF-100, length 10-30 $\mu \mathrm{m}$, diameter 50-200 nm, purity $>95 \%$ ) were purchased from Carbon Nano-material Technology (Korea); Methyl group-terminated PDMS
(MEP) which has properties [Serasense sf5, viscosity $5 \mathrm{mPa}^{*}$ s, relative density 0.95 , specific gravity 0.915 $\left.\left(25^{\circ} \mathrm{C}\right)\right]$ was purchased from KCC Corporation (Korea); and biocompatible PDMS [MED-6215, viscosity $3800 \mathrm{mPa} * \mathrm{~s}$, specific gravity $\left.1.03\left(25^{\circ} \mathrm{C}\right)\right]$ and adhesive PDMS (MG 7-9850, viscosity $2900 \mathrm{mPa} * \mathrm{~s}$, adhesion $1.1 \mathrm{~N} / 2.5 \mathrm{~cm}$ ) were purchased from Avantor ${ }^{\mathrm{TM}}$ (USA) and Dow Corning (USA), respectively.

\subsection{CNF Dispersion in PDMS}

To fabricate the dry electrode parts, CNFs and MEP were dispersed in biocompatible PDMS (MED-6215). To optimize the electrical performance and diffusion degree of uniformity, we developed a unique mixing and baking process (Fig. 1). First, CNFs of different mixing ratios were poured in isopropyl alcohol (IPA) and mixed in a vortex shaker (VORTEX 1, IKA ${ }^{\circledR}$ ) for 5 min. It is difficult for CNFs to be dispersed in biocompatible PDMS due to the high viscosity of PDMS (viscosity: $3800 \mathrm{mPa}^{*} \mathrm{~s}$ ). To solve this problem, MEP (viscosity: $5 \mathrm{mPa}^{*} \mathrm{~s}$ ) was poured in dispersed CNF/IPA and mixed in a vortex shaker for $5 \mathrm{~min}$. Then, MED-6215 part $\mathrm{A}$ was added to the mixing solution and stirred on a hot plate at $100{ }^{\circ} \mathrm{C}$ until the IPA was completely evaporated from the mixing solution. Finally, MED-6215 part B was added in a mass ratio of 1:10 and stirred with sticks. Dry electrodes of diverse sizes and thicknesses, were prepared by pouring in a prepared mold and baking on a hot plate. For ease of demolding, the molds were immersed under methanol for $1 \mathrm{~min}$; the methanol spread into small gaps, enabling separation [34]. The specific quantitative volume show below Table 1 .

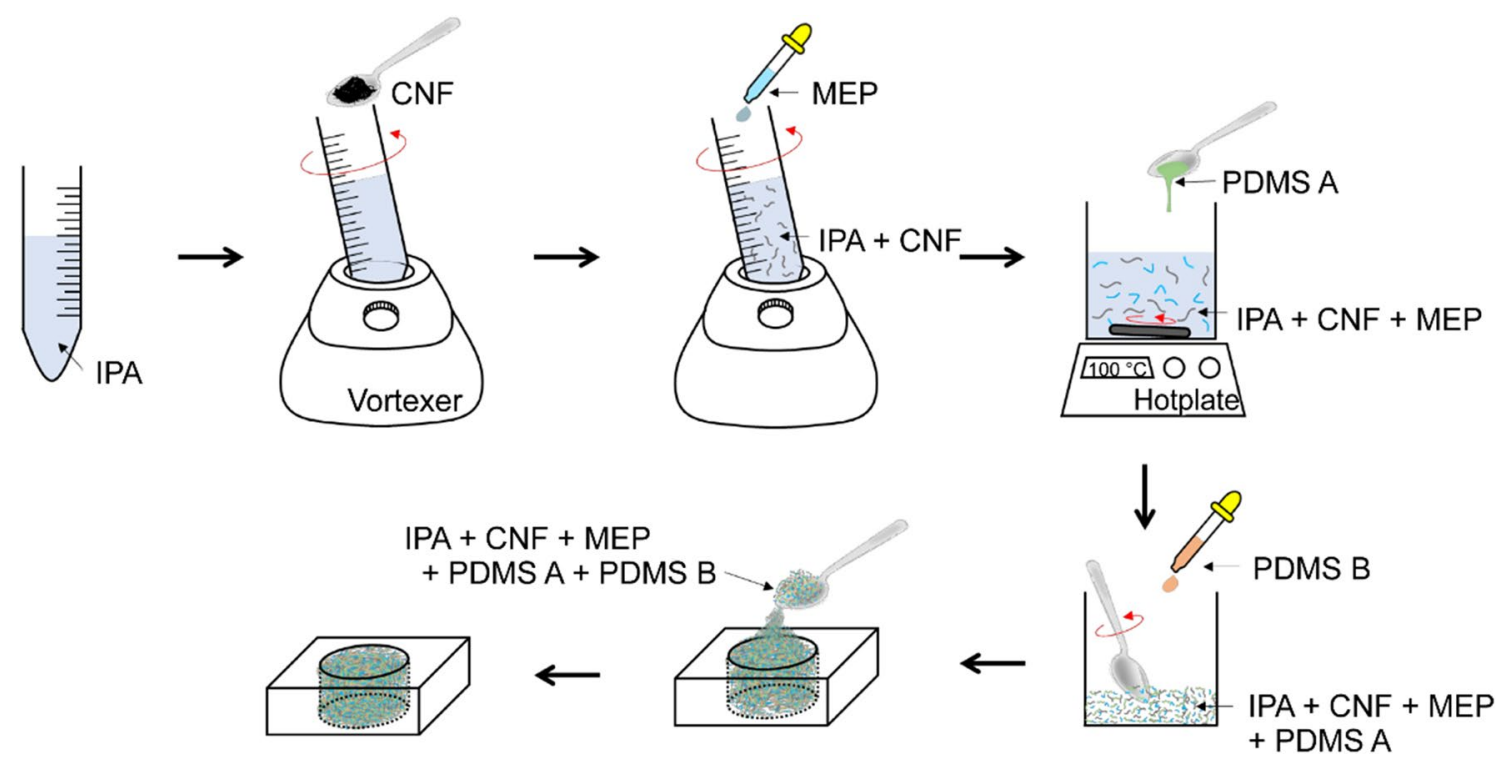

Fig. 1 Schematic diagram showing the process of CNF dispersal and dry electrode fabrication 
Table 1 Specific quantitative volume

\begin{tabular}{llllll}
\hline$w t \%$ & CNF (g) & IPA (g) & MEP (g) & PDMS A (g) & PDMS B (g) \\
\hline 15 & 9.5 & 30 & 10 & 40 & 4 \\
20 & 13.5 & 30 & 10 & 40 & 4 \\
25 & 18 & 30 & 10 & 40 & 4 \\
30 & 23.2 & 30 & 10 & 40 & 4 \\
\hline
\end{tabular}

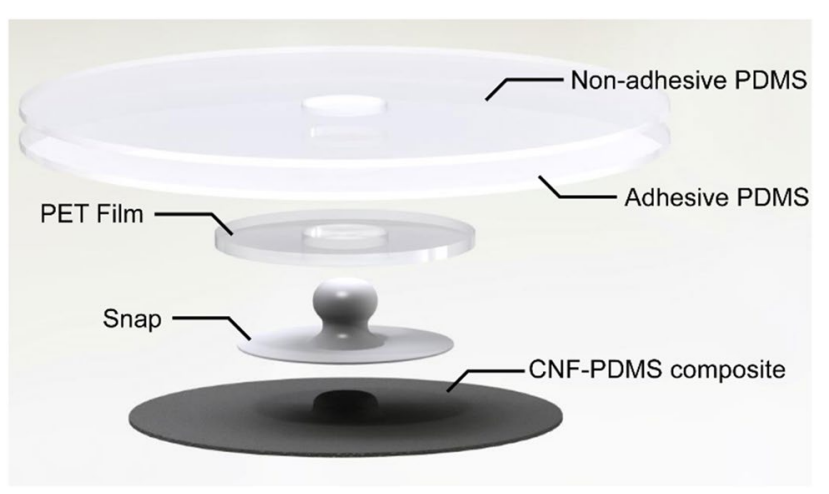

Fig. 2 Schematic of wearable patches

\subsection{Fabrication of Wearable Patch}

The wearable patch was assembled from two components: the dry electrode part for recording bio signals and the sticky patch part for mounting on the skin. The dry electrodes consisted of PDMS containing dispersed CNFs. The sticky patch parts were made by patterning and detaching from a smooth surface substrate after spin-coating and baking two types of PDMS. Non-adhesive PDMS (MED-6215) was spin coated on a glass wafer at $1000 \mathrm{rpm}$ for $30 \mathrm{~s}$ and baked on a hotplate at $100{ }^{\circ} \mathrm{C}$ for $1 \mathrm{~min}$. Then, adhesive PDMS (MG79850) was spin coated and baked under the same conditions. During this process, the attachable and detachable strengths of the sticky patch were measured and optimized for use in a wearable monitoring system. The two manufactured components were aligned and a hole was made in the center of the sticky component. For easy and repeated connection with a conventional signal processing system without any adapters, we inserted a conventional snap and polyester (PET) film into the hole. The construction of the wearable patches is shown in Fig. 2.

\subsection{Characterization of Thermal Properties}

To confirm thermal stability of the PDMS while heating process for fabricate dry electrode, we carried out the thermal gravimetric analysis (TGA). TGA of samples (weight: approximately 5-6 mg) was performed on a TGA Q50 (TA Instruments) under $\mathrm{N}_{2}$ gas atmosphere at a flow rate of $10 \mathrm{~mL} / \mathrm{min}$ using platinum pans. Temperature profiles ranged from 25 to $400{ }^{\circ} \mathrm{C}$ with a heating rate of $10^{\circ} \mathrm{C} / \mathrm{min}$.

\subsection{Characterization of Electrical Properties}

The sheet resistivity of the prepared samples was measured to evaluate the effect on electrical properties of CNF composition, thickness, and contact area. The CNF weight fraction and the contact area of the electrodes are thought to be critical factors determining the electrical properties. Fabricated $\mathrm{CNF} / \mathrm{PDMS}$ electrodes are flexible, stretchable, and have no fixed shape, in contrast to commercial ECG electrodes (Ag/ $\mathrm{AgCl})$. Therefore, we measured the sheet resistivity according to different CNF ratios to investigate the effect of weight fraction.

\subsection{Characterization of Electromechanical Properties}

To evaluate the electromechanical properties of dry electrodes, the electrodes were tested under cyclic load conditions. Specimens with dimensions of $20 \mathrm{~mm} \times 40 \mathrm{~mm} \times 1 \mathrm{~mm}$ were prepared with stretching area dimensions of $20 \mathrm{~mm} \times 20 \mathrm{~mm} \times 1 \mathrm{~mm}$. Prepared samples were fixed on a homemade motorized stretching machine and electrical resistivity was measured using a digital multimeter (Keysight 34410A) and a data acquisition program (Keysight BenchVue). The stretching length of the specimen was 1-6 mm (approximately 5-30\%), and the electrical resistivity was measured in situ (sampling rate: $120 \mathrm{~Hz}$ ). The hysteresis performance and strain profile of the dry electrodes were measured by incremental stretching (stretching speed: $1 \mathrm{~mm} / \mathrm{s}$ ). After stretching and releasing at each degree of strain $(5,10,15,20,25,30 \%)$, the apparatus was stopped for $15 \mathrm{~s}$ to relax the dry electrodes. The long-term durability of the dry electrodes were also tested by measuring the electrical resistivity sequentially over 10,000 cycles (stretching speed: $3 \mathrm{~mm} / \mathrm{s}$ ).

\subsection{ECG Measurement}

To acquire an ECG signal using the wearable patch, patches were placed on both wrists and the left ankle of human subjects. The ECG signal was amplified and acquired through a data acquisition system (BIOPAC systems MP36). Fabricated patches were evaluated through the measurement of ECG signals. These tests were performed for $60 \mathrm{~s}$ at a rate of 200 samples per second. To decrease the noise induced by the extension and movement cable of the ECG amplifier, cables from patches were fixed onto examinee's wrists and ankles using compression bandages. 


\subsection{Biocompatibility Tests}

L-929 (Mouse Fibroblasts) cells were purchased from ATCC (USA) and cultured in a minimum essential medium supplemented with $10 \%$ fetal bovine serum (FBS) and $1 \%$ penicillin-streptomycin $(\mathrm{P} / \mathrm{S})$ in a humid incubator at $37{ }^{\circ} \mathrm{C}$ and $5 \% \mathrm{CO}_{2}$. In order to test the toxicity of the materials to cells, biocompatibility was verified by MTT test (ISO 10993-5:2009). To compare with conventional electrodes, we prepared both manufactured wearable patches and conventional electrodes. For these tests, confluent monolayer cells were adjusted to a density of $1 \times 10^{5}$ cells $/ \mathrm{mL}$, and $0.1 \mathrm{~mL}$ were added to each of 96 well plates. The wells were incubated for $24 \mathrm{~h}$ to obtain an $80 \%$ confluent monolayer of cells. The growth medium was discarded from the wells and replaced with $2 \mathrm{~mL}$ of the reagent control, negative control, positive control, and test sample. The MTT test measures the degree of cell growth at an absorbance of $570 \mathrm{~nm}$. A decrease in number of living cells results in a decrease in the metabolic activity in the sample. This decrease directly correlates to the amount of blue-violet formazan formed, as monitored by the optical density at $570 \mathrm{~nm}$. The lower the value of Viab.\%, the higher the cytotoxic potential of the test sample. If viability is reduced to $<70 \%$ of the blank, it is judged to have cytotoxic potential.

The skin compatibility test was conducted on subjects wearing the wearable patch on their forearm for 7 days. During this time, observations were conducted of the side effects on the skin. To prevent inconvenience to the volunteer in their daily life, such as during bathing and exercise, a compression band was used to secure the wearable patch in case of necessity.

\section{Results and Discussion}

CNFs showed excellent dispersion properties in the PDMS prepolymer, and their mixing ratio was controlled as intended. Using the well-dispersed CNFs in PDMS, the fabrication of dry electrode parts was successfully performed by the processes described. Figure 3 illustrates a set of fabricated wearable patches; from left to right, the ratio of CNFs increased and the thickness was effectively controlled by the proposed replication method.

We obtained plot of the TGA mass loss curves to confirm thermal stability of PDMS while fabricate dry electrode (Fig. 4). These showed the typical thermal degradation curve

Fig. 3 Image of four fabricated types of wearable patch (left to right, $15,20,25$, and $30 \mathrm{wt} \%$ )

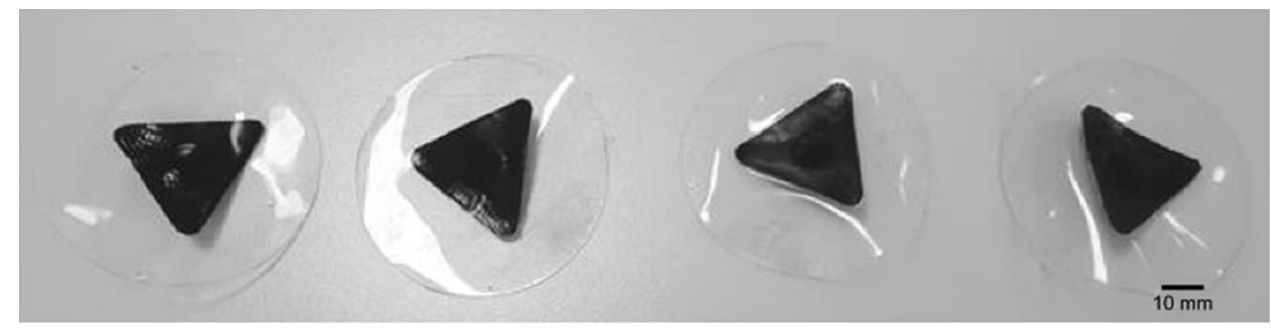

(a)

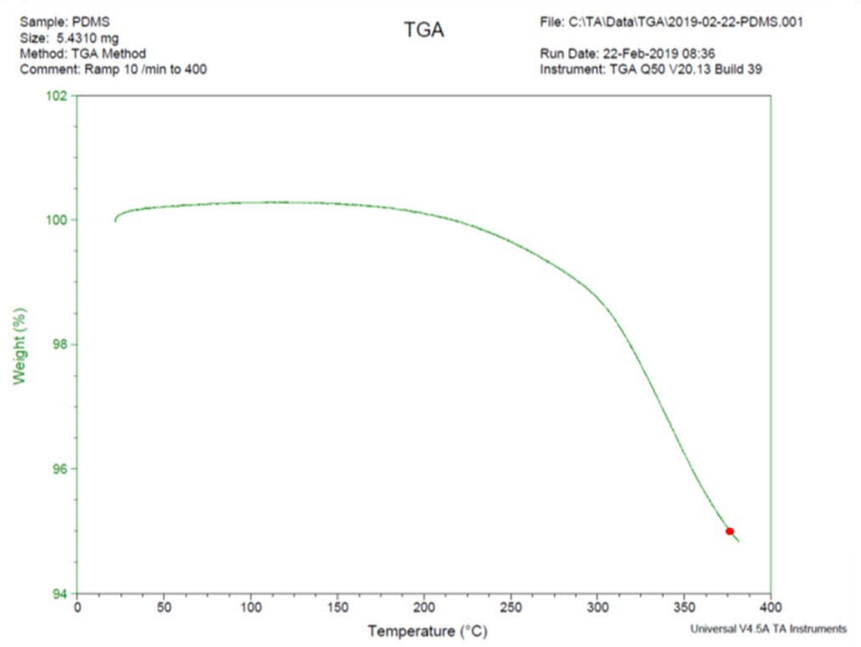

(b)

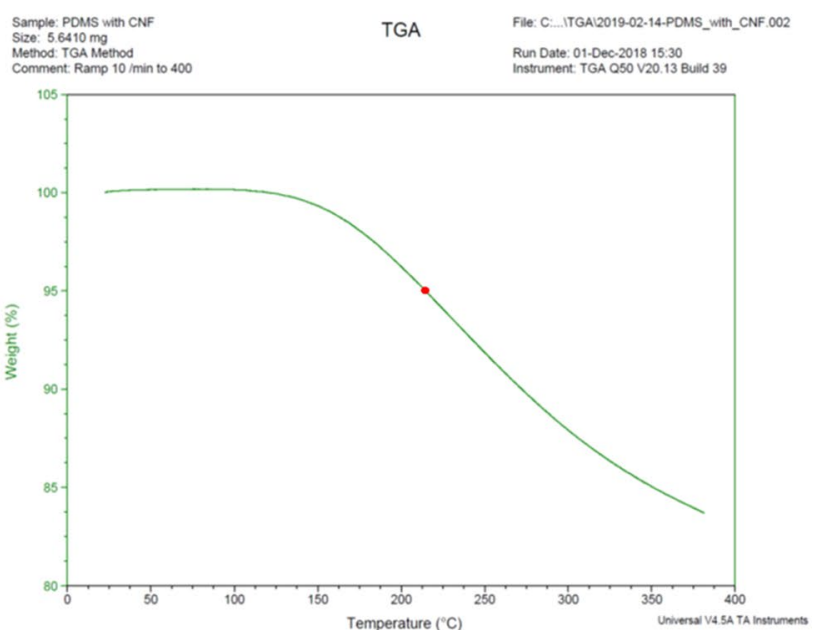

Fig. 4 TGA results for PDMS (a) and CNF/PDMS (b). (Red dot indicate $\mathrm{Td}_{5 \%}$ ) 
of PDMS and $\mathrm{Td}_{5 \%}$ (the temperature at $5 \%$ weight loss) are shown around $210^{\circ} \mathrm{C}(\mathrm{CNF} / \mathrm{PDMS}), 375^{\circ} \mathrm{C}$ (PDMS). These results can explain PDMS withstand the process temperature $\left(100{ }^{\circ} \mathrm{C}\right)$.

The sheet resistivity of the four fabricated sets of CNF/ PDMS composite $(15,20,25$, and $30 \mathrm{wt} \%$ ) was analyzed, and their thickness and diameter effects were also evaluated (Fig. 5). It must be noted that if a value above $30 \mathrm{wt} \%$ is used then the surface of the electrode becomes too rough; and if the less than $15 \mathrm{wt} \%$ is used, then the electrical conductivity becomes too low.

To more clearly observe the difference, we fabricated circular samples. As expected, the sheet resistivity decreased with an increase in CNF weight fraction and thickness and diameter of the dry electrode. However, the thickness and diameter effects were almost negligible compared to the $\mathrm{CNF}$ weight fraction. For a small diameter dry electrode, the change ratio of resistivity is larger than for a large diameter dry electrode. Also, no ECG signal was recorded at a CNF ratio of $<25 \mathrm{wt} \%$ ratio in the PDMS electrode.

Figure 6 shows the relative change in resistivity $\left(R / R_{0}\right)$ against the applied strain for two types of electrodes $(25$ and $30 \mathrm{wt} \%$ ). In this experiment, the CNF/PDMS specimens were well stretched, and the relative change in resistivity was not significant until 30\% stretching. Moreover, the response to applied strain shows good linearity. The $20 \mathrm{wt} \%$ specimen data were not included in Fig. 5 because of not showing clear differences during stretching and too much noise.

The elastic force and electrical resistivity of the dry electrode increased after decreasing the ratio of CNFs. Figure 7 presents the hysteresis performance of dry electrodes at different degrees of strain. The electrical resistivity of the dry electrodes was almost recovered after 30\% stretching and releasing, demonstrating clear differences at each strain.

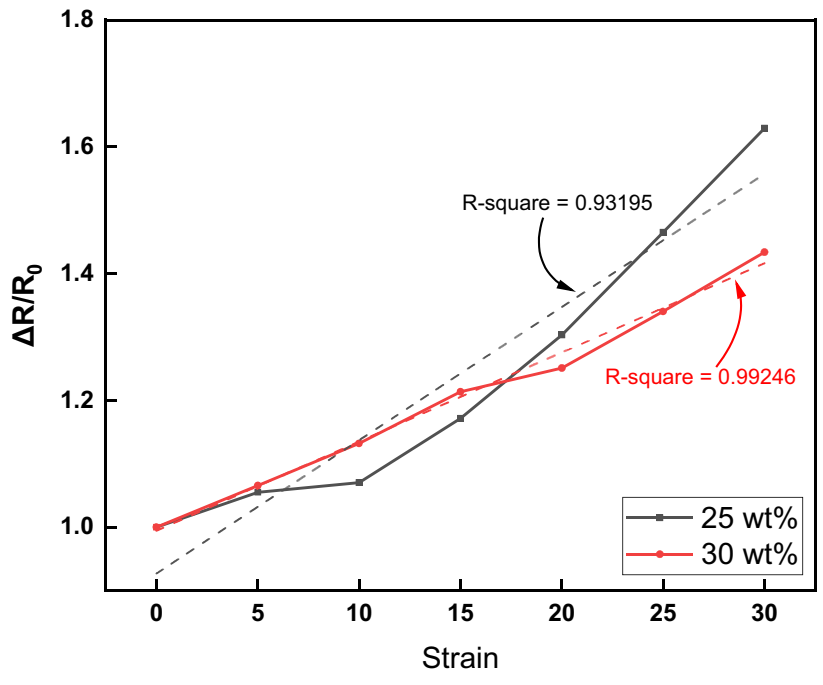

Fig. 6 Influence of strain on the change ratio of resistivity

However, short amounts of stretching and releasing at each strain led to sharp increases in the electrical resistance. This trend occurs because the CNFs rearrange in the PDMS elastomers under cyclic load conditions and the electrical resistance is restored with time.

Long-term durability tests were also performed at three degrees of strains $(10,20$, and 30\%, Fig. 8). All three samples showed excellent stability and recoverability over more than 10,000 cycles. The maximum relative change in resistance was 1.3, 2.7, and 3.1 at 10, 20, and 30\% strain, respectively.

Conventional $\mathrm{Ag} / \mathrm{AgCl}$ electrodes and fabricated wearable patches were prepared ( $25 \mathrm{wt} \% \mathrm{CNF}$ ratio, $2 \mathrm{~mm}$ thickness, $3 \mathrm{~cm}$ diameter) and ECG signals were measured. The (a)

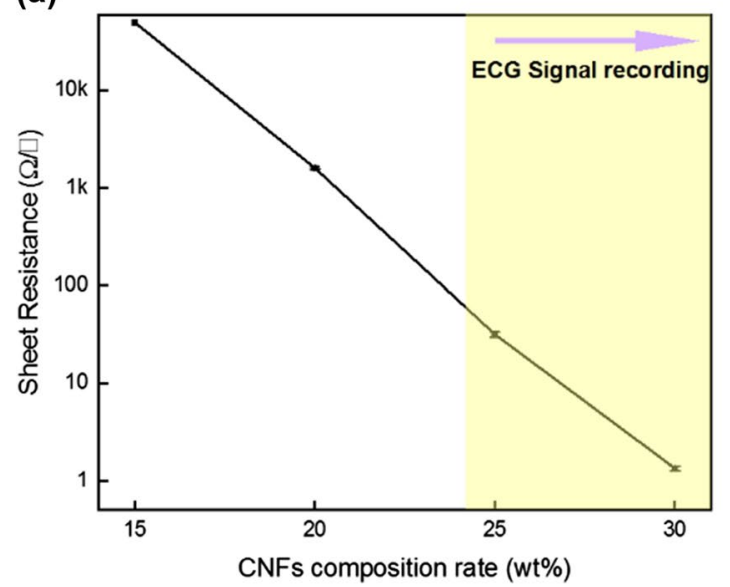

(b)

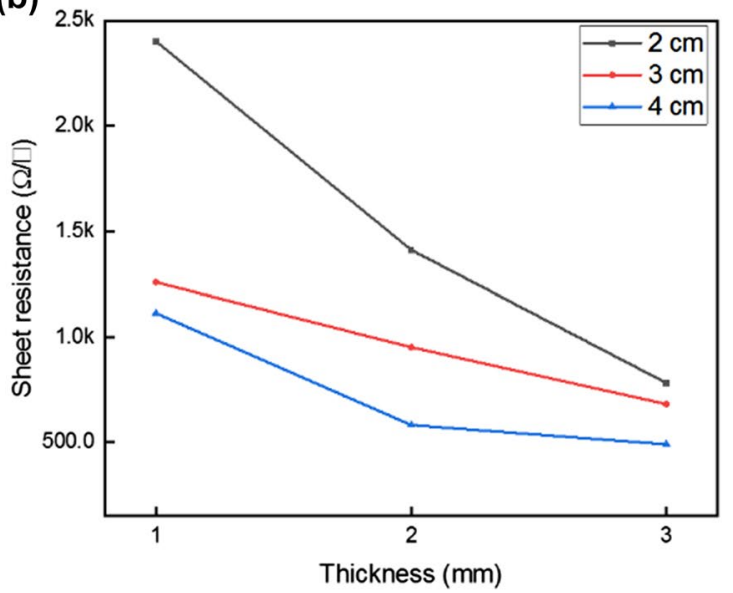

Fig. 5 Sheet resistivity of dry electrodes. a Sheet resistivity as a function of the CNF weight fraction. (3 cm diameter, $2 \mathrm{~mm}$ thickness electrodes), b Sheet resistivity as a function of the thickness and diameter of the dry electrode ( $30 \mathrm{wt} \%$ electrodes) 
(a)

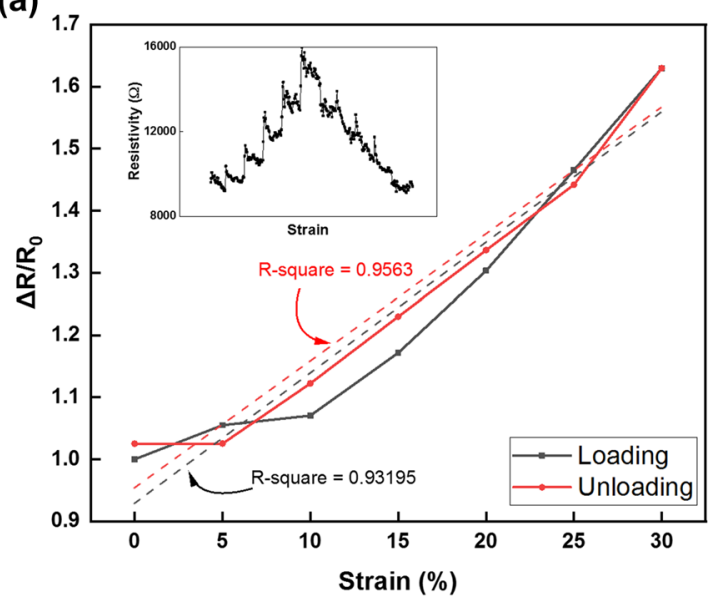

(b)

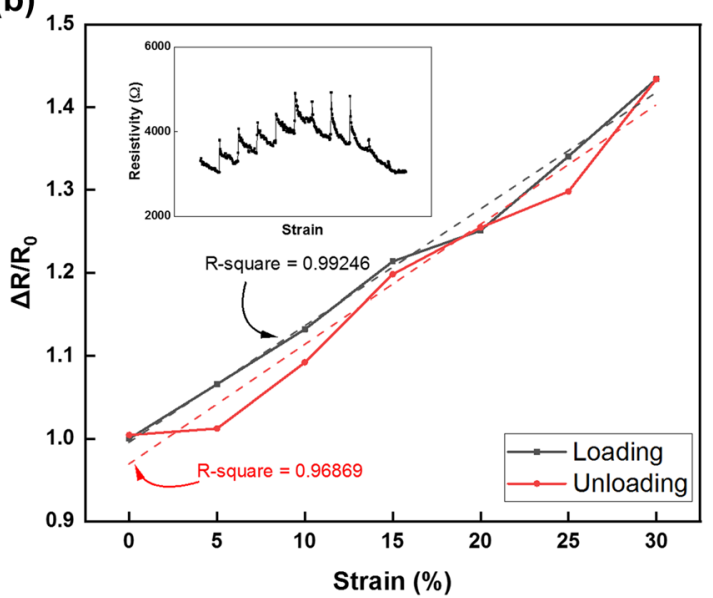

Fig. 7 Hysteresis performance of dry electrodes at different strain levels. a $25 \mathrm{wt} \%$ CNF dry electrodes. b $30 \mathrm{wt} \%$ CNF dry electrodes. Inset shows the strain profile

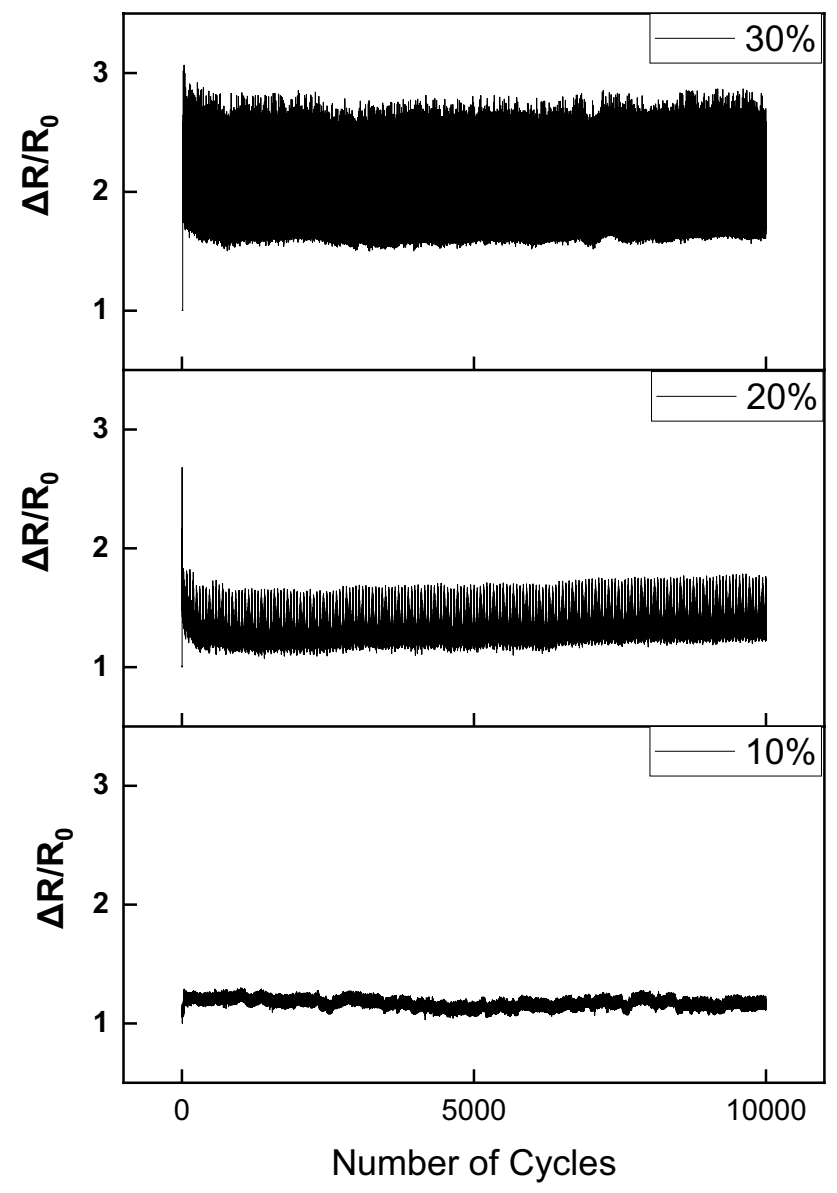

Fig. 8 Long-term durability test of dry electrodes over more than 10,000 cycles typical ECG waves from each electrode are illustrated in Fig. 9. The quality of the ECG signal measured using PDMS electrodes with over $25 \mathrm{wt} \%$ CNFs remained similar despite changing thickness and diameter. Moreover, PQRST waves were clearly shown in this figure. The biocompatibility test was performed by MTT assay on the wearable patch (CNF/ PDMS).

Conventional $\mathrm{Ag} / \mathrm{AgCl}$ electrodes and a negative control were also analyzed to compare the relative levels of cell viability. As shown in Fig. 10, the viability of L-929 cells in the negative control and CNF/PDMS electrode after $48 \mathrm{~h}$ was $94 \%$ and $75.1 \%$, respectively. Moreover, the cells on the CNF/PDMS electrode were alive and spread uniformly on the surface of the electrode. However, cell viability on the $\mathrm{Ag} / \mathrm{AgCl}$ electrode was significantly lower than on the CNF/PDMS electrode and the cell condition was completely destroyed. We found that cell proliferation was clearly higher on the CNF/PDMS electrode after $48 \mathrm{~h}$. These results reveal that CNFs and PDMS do not affect cells.

The skin compatibility test was conducted over 7 days. The skin under the electrode was normal and no itching or erythema were observed after 7 days of wear (Fig. 11).

\section{Conclusion}

In this study, we fabricated a simple carbon nanofiber-based wearable patch. The fabricated wearable patch was stretchable, skin-mountable, and biocompatible. The wearable patch was tested on three test subjects. The wearable patch showed potential for long-term measurement of ECG signals. The analysis of electrical characteristics showed that the ratio of CNFs is a key factor influencing electrical performance and ECG signal detection. In the ECG test, even though 


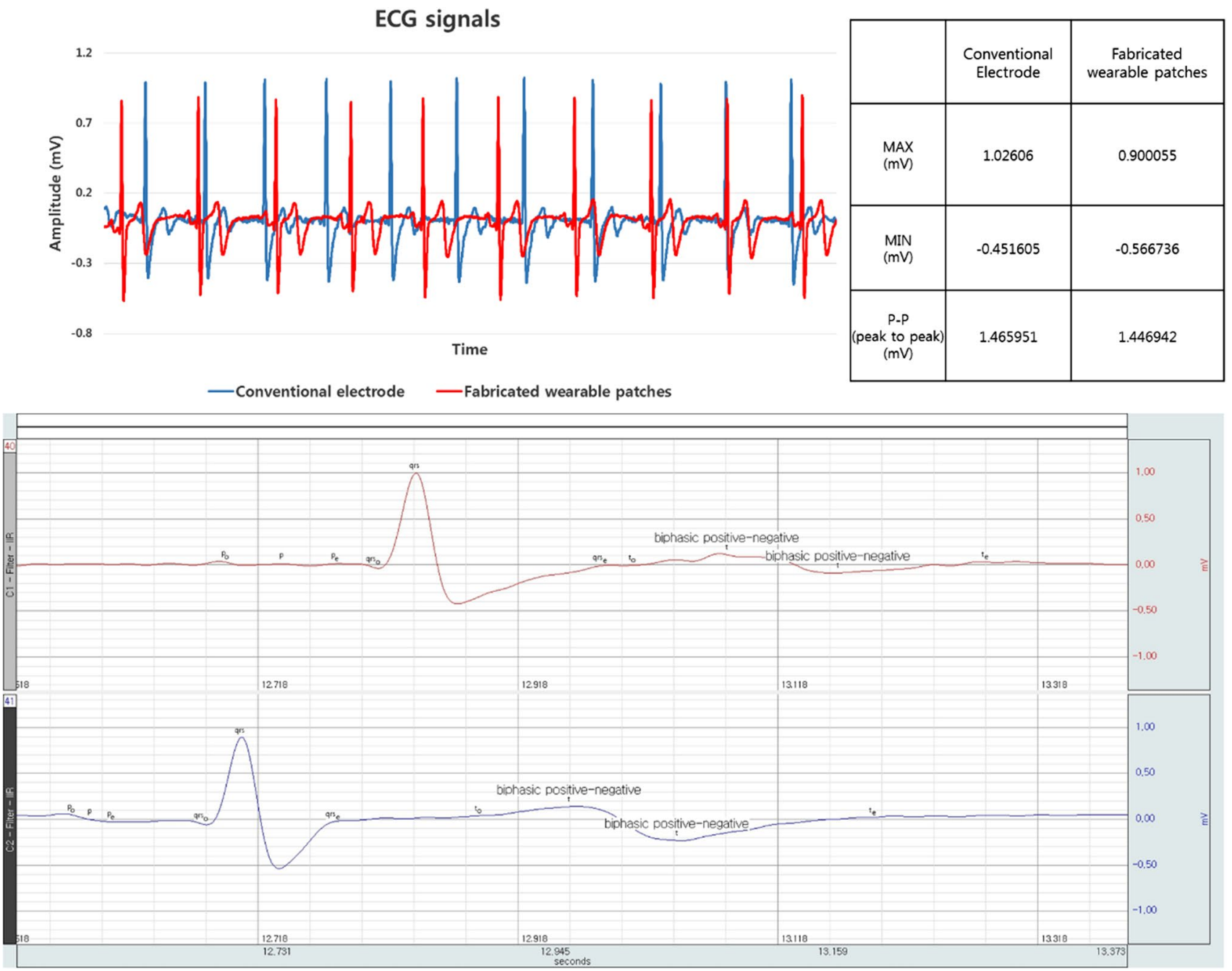

Fig. 9 Measured ECG signals from fabricated wearable patches and conventional electrodes

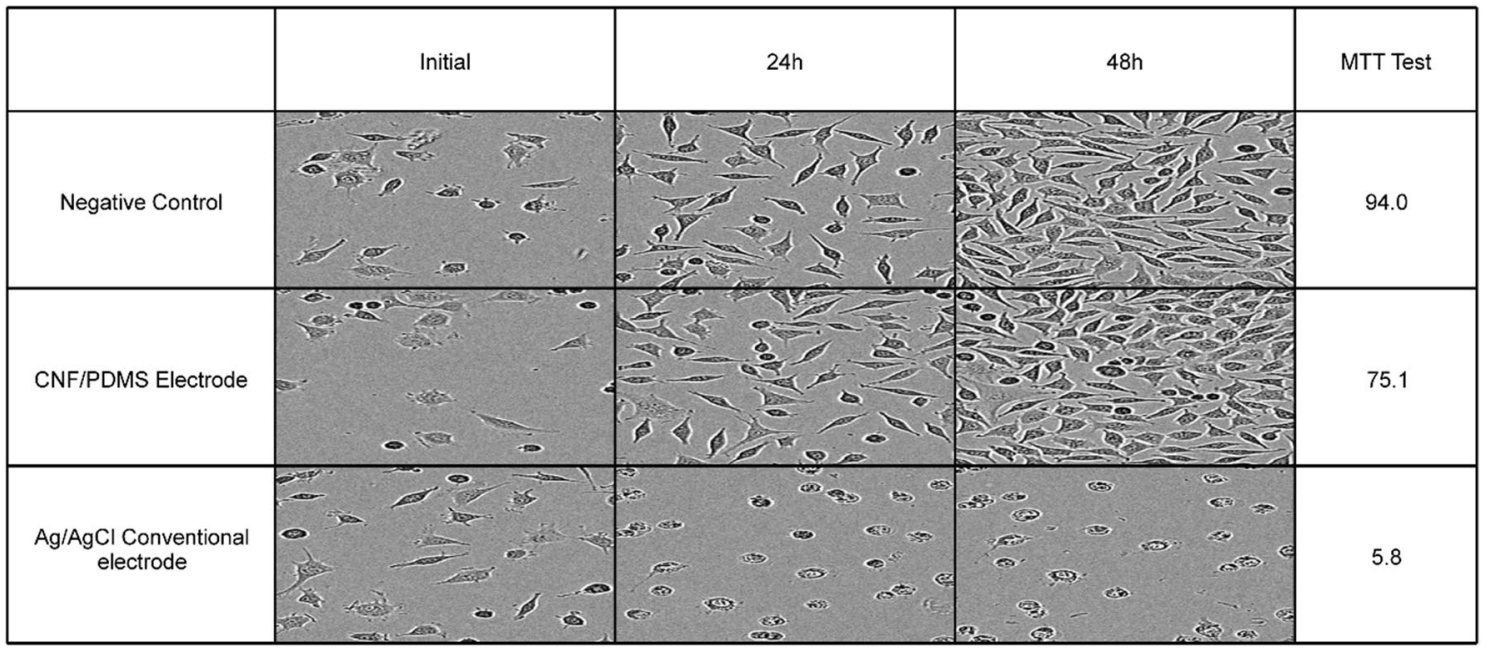

Fig. 10 Results of the biocompatibility test 
Fig. 11 Results of the long-term (7 days) skin compatibility test. a Image of wearing electrode on the skin. b Image of the skin after detaching electrode

\section{(a)}

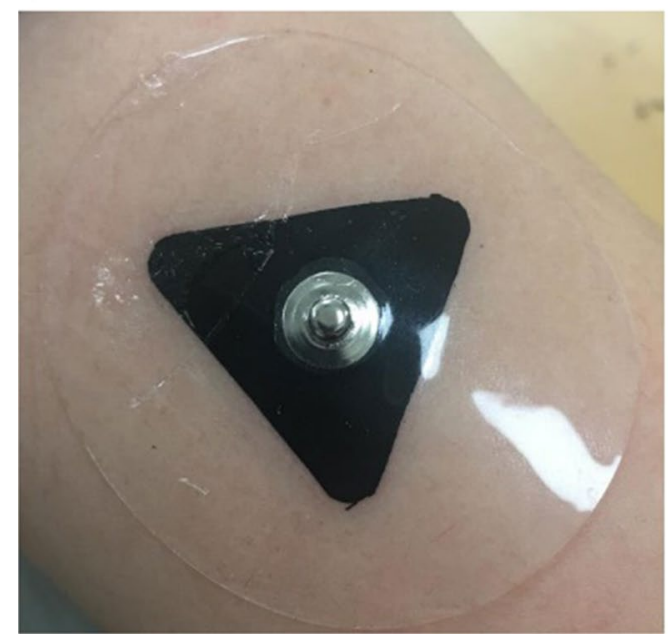

(b)

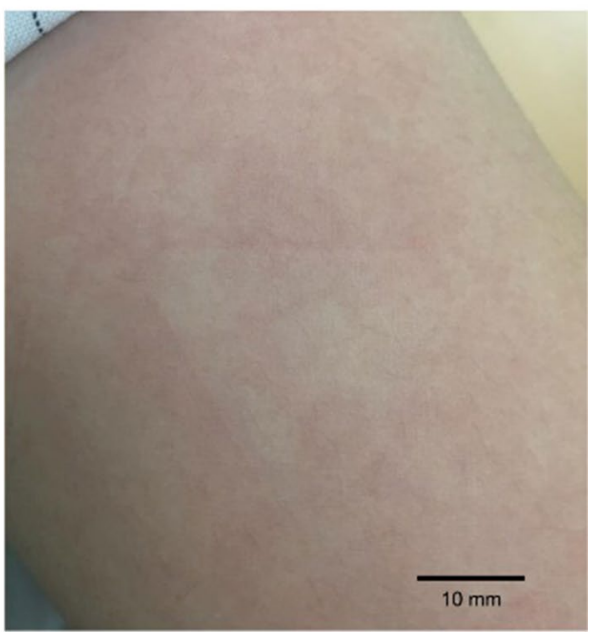

the amplitude was slightly lower than that of the $\mathrm{Ag} / \mathrm{AgCl}$ electrode, PQRST waves were clearly visible and baselines were stable. In addition, we discovered that our electrode has no side effects such as itching or irritation even after 1 week of continuous wear. We expect that the proposed CNF/PDMS composite electrode has important applications for daily, universal, and mobile health care technology. The fabricated dry electrode may have other useful applications including strain, pressure, and temperature gauges in a variety of bio-signal monitoring equipment.

Acknowledgements This work was supported by the National Research Foundation of Korea (NRF) Grant funded by the Korean Government (MSIP) Grant No. [NRF-2016R1A5A1921651].

\section{Compliance with Ethical Standards}

Conflicts of interest The authors declare that they have no conflict of interest.

Open Access This article is distributed under the terms of the Creative Commons Attribution 4.0 International License (http://creativeco mmons.org/licenses/by/4.0/), which permits unrestricted use, distribution, and reproduction in any medium, provided you give appropriate credit to the original author(s) and the source, provide a link to the Creative Commons license, and indicate if changes were made.

\section{References}

1. Patel, S., Park, H., Bonato, P., Chan, L., \& Rodgers, M. (2012). A review of wearable sensors and systems with application in rehabilitation. Journal of NeuroEngineering and Rehabilitation, 9(1), 21. https://doi.org/10.1186/1743-0003-9-21.

2. Kim, T., Park, J., Sohn, J., Cho, D., \& Jeon, S. (2016). Bioinspired, highly stretchable, and conductive dry adhesives based on 1D-2D hybrid carbon nanocomposites for all-in-one ECG electrodes. ACS
Nano, 10(4), 4770-4778. https://doi.org/10.1021/acsnano.6b013 55.

3. Lorwongtragool, P., Sowade, E., Watthanawisuth, N., Baumann, R. R., \& Kerdcharoen, T. (2014). A novel wearable electronic nose for healthcare based on flexible printed chemical sensor array. Sensors (Switzerland), 14(10), 19700-19712. https://doi. org/10.3390/s141019700.

4. Liu, Y., Norton, J. J. S., Qazi, R., Zou, Z., Ammann, K. R., Liu, H., et al. (2016). Epidermal mechano-acoustic sensing electronics for cardiovascular diagnostics and human-machine interfaces. Science Advances, 2(11), e1601185-e1601185. https://doi.org/10.1126/ sciadv. 1601185 .

5. Klingeberg, T., \& Schilling, M. (2012). Mobile wearable device for long term monitoring of vital signs. Computer Methods and Programs in Biomedicine, 106(2), 89-96. https://doi. org/10.1016/j.cmpb.2011.12.009.

6. Jeong, J. W., Kim, M. K., Cheng, H., Yeo, W. H., Huang, X., Liu, Y., et al. (2014). Capacitive epidermal electronics for electrically safe, long-term electrophysiological measurements. Advanced Healthcare Materials, 3(5), 642-648. https://doi.org/10.1002/ adhm.201300334.

7. Kim, J., Salvatore, G. A., Araki, H., Chiarelli, A. M., Xie, Z., Banks, A., et al. (2016). Battery-free, stretchable optoelectronic systems for wireless optical characterization of the skin. Science Advances, 2(8), e1600418-e1600418. https://doi.org/10.1126/ sciadv. 1600418.

8. Kim, J., Son, D., Lee, M., Song, C., Song, J. K., Koo, J. H., et al. (2016). A wearable multiplexed silicon nonvolatile memory array using nanocrystal charge confinement. Science Advances, 2(1), e1501101. https://doi.org/10.1126/sciadv.1501101.

9. Trung, T. Q., \& Lee, N. E. (2016). Flexible and stretchable physical sensor integrated platforms for wearable human-activity monitoringand personal healthcare. Advanced Materials, 28(22), 4338-4372. https://doi.org/10.1002/adma.201504244.

10. Oresko, J. J., Jin, Z., Cheng, J., Huang, S., Sun, Y., Duschl, H., et al. (2010). A wearable smartphone-based platform for real-time cardiovascular disease detection via electrocardiogram processing. IEEE Transactions on Information Technology in Biomedicine, 14(3), 734-740. https://doi.org/10.1109/TITB.2010.2047865.

11. Zheng, Y. L., Ding, X. R., Poon, C. C. Y., Lo, B. P. L., Zhang, H., Zhou, X. L., et al. (2014). Unobtrusive sensing and wearable devices for health informatics. IEEE Transactions on 
Biomedical Engineering, 61(5), 1538-1554. https://doi. org/10.1109/tbme.2014.2309951.

12. Choi, S., Lee, H., Ghaffari, R., Hyeon, T., \& Kim, D. H. (2016). Recent advances in flexible and stretchable bio-electronic devices integrated with nanomaterials. Advanced Materials, 28(22), 4203-4218. https://doi.org/10.1002/adma.201504150.

13. Takei, K., Honda, W., Harada, S., Arie, T., \& Akita, S. (2015). Toward flexible and wearable human-interactive health-monitoring devices. Advanced Healthcare Materials, 4(4), 487-500. https ://doi.org/10.1002/adhm.201400546.

14. Lee, H., Choi, T. K., Lee, Y. B., Cho, H. R., Ghaffari, R., Wang, L., et al. (2016). A graphene-based electrochemical device with thermoresponsive microneedles for diabetes monitoring and therapy. Nature Nanotechnology, 11(6), 566-572. https://doi. org/10.1038/nnano.2016.38.

15. Barro, S., Ruiz, R., Cabello, D., \& Mira, J. (1989). Algorithmic sequential decision-making in the frequency domain for life threatening ventricular arrhythmias and imitative artefacts: A diagnostic system. Journal of Biomedical Engineering, 11(4), 320-328. https://doi.org/10.1016/0141-5425(89)90067-8.

16. Searle, A., \& Kirkup, L. (2000). A direct comparison of wet, dry and insulating bioelectric recording electrodes. Physiological Measurement, 21(2), 271-283. https://doi. org/10.1088/0967-3334/21/2/307.

17. Myers, A. C., Huang, H., \& Zhu, Y. (2015). Wearable silver nanowire dry electrodes for electrophysiological sensing. RSC Advances, 5(15), 11627-11632. https://doi.org/10.1039/C4RA1 $5101 \mathrm{~A}$.

18. Kabiri Ameri, S., Ho, R., Jang, H., Tao, L., Wang, Y., Wang, L., et al. (2017). Graphene electronic tattoo sensors. ACS Nano, 11(8), 7634-7641. https://doi.org/10.1021/acsnano.7b02182.

19. Peng, H. L., Liu, J. Q., Dong, Y. Z., Yang, B., Chen, X., \& Yang, C. S. (2016). Parylene-based flexible dry electrode for bioptential recording. Sensors and Actuators B, 231, 1-11. https://doi. org/10.1016/j.snb.2016.02.061

20. Liu, B., Luo, Z., Zhang, W., Tu, Q., \& Jin, X. (2016). Carbon nanotube-based self-adhesive polymer electrodes for wireless long-term recording of electrocardiogram signals. Journal of Biomaterials Science, Polymer Edition, 27(18), 1899-1908. https:// doi.org/10.1080/09205063.2016.1239951.

21. Bihar, E., Roberts, T., Ismailova, E., Saadaoui, M., Isik, M., Sanchez-Sanchez, A., et al. (2017). Fully printed electrodes on stretchable textiles for long-term electrophysiology. Advanced Materials Technologies, 2(4), 1600251. https://doi.org/10.1002/ admt.201600251.

22. Gao, W., Emaminejad, S., Nyein, H. Y. Y., Challa, S., Chen, K., Peck, A., et al. (2016). Fully integrated wearable sensor arrays for multiplexed in situ perspiration analysis. Nature, 529(7587), 509-514. https://doi.org/10.1038/nature16521.
23. Yao, S., Myers, A., Malhotra, A., Lin, F., Bozkurt, A., Muth, J. F., et al. (2017). A wearable hydration sensor with conformal nanowire electrodes. Advanced Healthcare Materials, 6(6), 2524. https://doi.org/10.1002/adhm.201601159.

24. Agar, J. C. (2011). Highly conductive stretchable electrically conductive composites for electronic and radio frequency devices (Doctoral dissertation, Georgia Institute of Technology, 2011).

25. Khosla, A. (2012). Nanoparticle-doped electrically-conducting polymers for flexible nano-micro systems. Interface Magazine, 21(3-4), 67-70. https://doi.org/10.1149/2.F04123-4if.

26. Gigon, A., \& Shaarawy, T. (2016). The suprachoroidal route in glaucoma surgery 1 medical student (final year). Journal of Current Glaucoma Practice, 1010(11), 13-20. https://doi. org/10.5005/jp-journals-10008-1197.

27. Lee, S.E., Jun, S.B., Kim, J.H., Min, K.S., Kim, J.H., Eom, K.S., Jeong, J.S., Chang, J.W., Kim, S.J., (2012). A compact deep brain stimulation system for animal experiments. In Proceedings of international functional electrical stimulation society, 9-12

28. Chłopek, J., Czajkowska, B., Szaraniec, B., Frackowiak, E., Szostak, K., \& Béguin, F. (2006). In vitro studies of carbon nanotubes biocompatibility. Carbon, 44(6), 1106-1111. https://doi. org/10.1016/j.carbon.2005.11.022.

29. Smart, S. K., Cassady, A. I., Lu, G. Q., \& Martin, D. J. (2006). The biocompatibility of carbon nanotubes. Carbon, 44(6), 10341047. https://doi.org/10.1016/j.carbon.2005.10.011.

30. Chowdhury, S., Olima, M., Liu, Y., \& Saha, M. (2017). Carbon nanofiber and pdms based nanocomposite with sensing. ASME 2016 international mechanical engineering congress and exposition, 1-6.

31. Chowdhury, S., Olima, M., Liu, Y., Saha, M., Bergman, J., \& Robison, T. (2016). Poly dimethylsiloxane/carbon nanofiber nanocomposites: Fabrication and characterization of electri$\mathrm{cal}$ and thermal properties. International Journal of Smart and Nano Materials, 7(4), 236-247. https://doi.org/10.1080/19475 411.2016.1269027.

32. Lee, S. A., Jung, H. C., Kwon, D. H., Kim, A. H., \& Moon, J. H. (2017). Conductive cottons for patch type wearable bio potential monitoring. BIOENG'17 Proceedings, 1, 76-80.

33. Wu, S., Zhang, J., Ladani, R. B., Ravindran, A. R., Mouritz, A. P., Kinloch, A. J., et al. (2017). Novel electrically conductive porous PDMS/carbon nanofiber composites for deformable strain sensors and conductors. ACS Applied Materials \& Interfaces, 9(16), 14207-14215. https://doi.org/10.1021/acsami.7b00847.

34. Kim, J. Y., Baek, J. Y., Lee, K. A., \& Lee, S. H. (2005). Automatic aligning and bonding system of PDMS layer for the fabrication of 3D microfluidic channels. Sensors and Actuators A, 119(2), 593-598. https://doi.org/10.1016/j.sna.2004.09.023. 